\title{
Fungos anamórficos (Hyphomycetes) da Chapada Diamantina: novos registros para o Estado da Bahia e Brasil
}

\author{
Alisson Cardoso Rodrigues da Cruz ${ }^{1,3}$, Marcos Fabio Oliveira Marques ${ }^{1,2}$ e \\ Luís Fernando Pascholati Gusmão ${ }^{1}$
}

Recebido em 30/09/2005. Aceito em 5/03/2007

\begin{abstract}
RESUMO - (Fungos anamórficos (Hyphomycetes) da Chapada Diamantina: novos registros para o Estado da Bahia e Brasil). Os fungos anamórficos, caracterizados pela produção de estruturas de reprodução assexuadas, são habitantes comuns do folhedo onde desempenham papel importante na decomposição. O objetivo deste trabalho foi realizar um inventário dos fungos anamórficos associados ao folhedo de plantas da Chapada Diamantina, BA. Foram realizadas 13 expedições, de dezembro/2002 a outubro/2003, para coleta de folhedo. Para verificação da presença de fungos anamórficos o material foi submetido à técnica de lavagem sucessiva com água destilada esterilizada e posteriormente incubado em câmaras-úmidas. Lâminas permanentes com as estruturas reprodutivas dos espécimes foram confeccionadas com resina PVL e depositadas no herbário HUEFS. Das 57 espécies de fungos anamórficos identificados, nove constituem novas ocorrências para o Estado da Bahia e cinco para o Brasil: Fusariella atrovirens (Berk.) Sacc., Kiliophora ubiensis (Caneva \& Rambelli) Kuthub. \& Nawawi, Paraceratocladium silvestre Castañeda, Pleurotheciopsis setiformis Castañeda e Triscelophorus deficiens (Matsush.) Matsush. Incluem-se comentários e distribuição geográfica dos novos registros para o Estado da Bahia; descrições e ilustrações são apresentadas para as novas ocorrências para o Brasil.
\end{abstract}

Palavras-chave: Biodiversidade, folhedo, taxonomia

\begin{abstract}
Anamorphic fungi (Hyphomycetes) from the Chapada Diamantina: new records from Bahia State and Brazil). The anamorphic fungi are characterized by production of asexual reproductive structures and are common inhabitants of the leaf litter, where they play an important role in decomposition. The aim of this work was to survey the anamorphic fungi associated with leaf litter from Chapada Diamantina, Bahia state. Thirteen expeditions took place from December/2002 to October/2003 to collect leaf litter. The serial washing technique with sterile distilled water followed by incubation in moist chambers was utilized to verify the presence of anamorphic fungi. Permanent slides of the reproductive structures of specimens were prepared with PVL resin and deposited at the HUEFS Herbarium. Of the 57 species of anamorphic fungi identified, nine represent new records in Bahia state, and five in Brazil, as follows: Fusariella atrovirens (Berk.) Sacc., Kiliophora ubiensis (Caneva \& Rambelli) Kuthub. \& Nawawi, Paraceratocladium silvestre Castañeda, Pleurotheciopsis setiformis Castañeda and Triscelophorus deficiens (Matsush.) Matsush. Comments and geographic distribution are included for the new records in Bahia; descriptions and illustrations of the new records in Brazil are presented.
\end{abstract}

Key words: Biodiversity, leaf litter, taxonomy

\section{Introdução}

O folhedo compõe a maior parte da matéria orgânica acumulada no solo e tem, nos fungos anamórficos, um dos principais agentes responsáveis pela reciclagem dos nutrientes (Meguro et al. 1979; Hudson 1968).

No Brasil, estudos sobre fungos anamórficos ainda são escassos. A partir da década de 90, os estudos tornaram-se mais frequientes, principalmente sobre Hyphomycetes decompositores. Dentre o material vegetal, o folhedo foi o mais investigado. A maior parte dos trabalhos foram realizados no Bioma Mata Atlântica do Estado de São Paulo, com destaque para Grandi \& Attili (1996), que identificaram 16 Hyphomycetes associados à Alchornea triplinervia (Spreng.) Müell. Arg., mencionando dois novos registros para o Brasil. Grandi (1999) coletou 26 espécies, sendo seis citadas pela primeira vez para o país. Gusmão et al. (2001) verificaram a presença de 55 espécies associadas à Miconia cabussu Hoehne, descrevendo 14 como novas ocorrências para o Brasil e propondo uma nova espécie para a ciência (Gusmão et al. 2000). Grandi \& Gusmão (2002) estudaram o folhedo de Tibouchina

\footnotetext{
1 Universidade Estadual de Feira de Santana, Departamento de Ciências Biológicas, Laboratório de Micologia, C. Postal 252, 44031-460 Feira de Santana, BA, Brasil (lgusmao@uefs.br)

2 Universidade do Estado da Bahia, Campus VII, Departamento de Educação, Laboratório de Microbiologia, Rodovia Lomanto Júnior, BR 407 km 127, 48970-000 Senhor do Bonfim, BA, Brasil (mfomarques@yahoo.com.br)

3 Autor para correspondência: alissonbio@yahoo.com.br
} 
pulchra Cogn. e verificaram a presença de 22 táxons, sendo três novos registros para o país.

Especificamente para a Bahia, A.C. Batista e colaboradores, nas décadas de 50 a 70 , coletaram 50 gêneros e 65 espécies de fungos anamórficos, dos quais cerca de $70 \%$ correspondem a Hyphomycetes e 30\% pertencem aos Coelomycetes (Silva \& Minter 1995). Após esse período, apenas no início do século XXI foram retomados os estudos dos fungos anamórficos no Estado. Gusmão \& Barbosa (2003) referiram pela primeira vez para o país, Paraceratocladium polysetosum Castañeda e relataram a conidiogênese polifialídica para o táxon. L. Gusmão, dados não publicados, descreveu e ilustrou 73 espécies de fungos anamórficos para os campos rupestres do Estado, sendo 22 espécies novas citações para o país e uma inédita para a ciência. Gusmão \& Barbosa (2005) descreveram Hemibeltrania malaysiana Matsush., e apresentaram uma chave para as espécies do gênero. Gusmão et al. (2005a) pesquisando os fungos anamórficos no semi-árido baiano, relataram sete espécies de Curvularia Boedijn. Barbosa \& Gusmão (2005) apresentaram duas espécies de Speiropsis Tubaki, sendo relatada $S$. pedatospora Tubaki pela primeira vez para o Brasil. Para a Chapada Diamantina, Gusmão et al. (2005b) registraram 57 espécies de fungos anamórficos. Recentemente Castañeda-Ruiz et al. (2006) descreveram duas novas espécies de Brachydesmiella G. Arnaud ex S. Hughes, além de apresentarem duas propostas de combinação e novas ocorrências de Hyphomycetes para o país.

Portanto, estudos taxonômicos e de biodiversidade de fungos anamórficos no Estado da Bahia são ainda insipientes. Este trabalho teve como objetivo inventariar os fungos anamórficos associados ao folhedo, visando o aumento do conhecimento sobre a diversidade fúngica da Chapada Diamantina.

\section{Material e métodos}

A Chapada Diamantina, porção norte da Cadeia do Espinhaço ocupa uma área de $38.000 \mathrm{~km}^{2} \mathrm{e}$ representa $15 \%$ do Estado da Bahia (Giulietti \& Pirani 1988). É composta, além do maciço central, mais contínuo, por afloramentos dispersos, especialmente ao norte como na região de Morro do Chapéu (Harley 1995). A diversidade de fisionomias na região inclui desde a caatinga, marcada pela baixa pluviosidade, até diversos tipos de florestas, como as matas ciliares e de planalto, culminando nas áreas mais elevadas com vegetações abertas, como cerrados e campos rupestres. Toda essa diversidade está sujeita à degradação por atividades antrópicas, como a abertura de pastagens, a exploração de recursos vegetais nativos e a agricultura (Harley 1995; Giulietti et al. 1996).

Foram realizadas 13 expedições na Chapada Diamantina, entre dezembro/2002 a outubro/2003, em sete áreas, localizadas nos municípios de Jussiape, Lençóis, Mucugê, Palmeiras e Rio de Contas, para coletas em quatro tipos vegetacionais: caatinga, campo rupestre, cerrado e mata semidecídua. As coordenadas geográficas dos pontos de coleta foram obtidas com o auxílio de GPS (Global Positioning System) Garmin GPS 12.

Folhas de várias espécies vegetais foram coletadas diretamente do folhedo, constituindo amostras compostas de vinte folhas, que foram submetidas à técnica de lavagem sucessiva de substrato e plaqueamento, descrita por Harley \& Waid (1955) e modificada por Grandi \& Gusmão (1998). Depois de lavadas, as folhas foram fragmentadas e dispostas em 10 câmaras-úmidas (placa de Petri + papel filtro umedecido), permanecendo em temperatura ambiente $\left(25^{\circ} \mathrm{C}\right)$ por 45 dias, para isolamento das estruturas reprodutivas. Lâminas permanentes foram confeccionadas com resina PVL (álcool polivinílico + lactofenol); para estruturas hialinas e de coloração com gradação do castanho, foi adicionado o corante azul de algodão (Trappe \& Schenck 1982). Para identificação, foram realizadas medições das estruturas de importância taxonômica e consultada a literatura especializada. Posteriormente as lâminas foram depositadas no Herbário da Universidade Estadual de Feira de Santana (HUEFS). Foram incluídos comentários e distribuição geográfica para os novos registros para a Bahia, além de descrições e ilustrações das novas ocorrências para o Brasil.

\section{Resultados e discussão}

Chloridium virescens var. virescens (Pers.) Gams \& Hol.-Jech., Stud. Mycol. 13: 17. 1976.

$\equiv$ Dematium virescens Pers., Tent. Dispos. meth. Fung. p. 41.1797.

$=$ Chloridium viride Link, Mag. Ges. naturf. Freunde, Berl. 3:13. 1809.

=Sporotrichum virescens Link, Mag. Ges. naturf. Freunde, Berl. 3:13. 1809.

=Doratomyces viridis Corda, Weitenwebers Beitr. ges. Nat.-Heilwiss. p. 83. 1836. 
$=$ Haplaria chlorina Ell. \& Ev., Bull. Torrey bot. Club 10: 97. 1883.

=Mesobotrys flavovirens Höhn., Sber. K. Akad. Wiss. Wien, Math.-nat. Kl. III: 1048. 1902.

Descrição e ilustração: Grandi \& Attili (1996).

A variedade estudada é comumente isolada de galhos e de diferentes tipos de madeira, em estágios avançados de decomposição (Gams \& HolubováJechová 1976). No Brasil, esta variedade foi pela primeira vez encontrada, sobre folhas de Alchornea triplinervia (Spreng.) Müell. Arg. no Estado de São Paulo (Grandi \& Attili 1996).

Material examinado: BRASIL. Bahia: Palmeiras, alto do Morro do Pai Inácio, campo rupestre, $\mathrm{S} 12^{\circ} 27^{\prime}$ e W41 $28^{\circ}, 17 / \mathrm{II} / 2003$, sobre folhas mortas, A.C.R. Cruz (HUEFS-61599).

Distribuição geográfica: Bélgica, Dinamarca, França, Grã-Bretanha, Holanda (Gams \& HolubováJechová 1976), Brasil (Grandi \& Attili 1996), China, Tailândia (Farr et al. 2007).

Cryptophiale kakombensis Piroz., Can. J. Bot. 46: 1124. 1968.

Descrição e ilustração: Grandi \& Attili (1996).

Espécie comum nas regiões tropicais e subtropicais (Mercado-Sierra et al. 1997; Grandi \& Gusmão 2002). Além desta, C. minor Farr e C. udagawae Piroz. \& Ichinoe já foram mencionadas no Brasil (Farr 1980; Grandi \& Attili 1996).

Material examinado: BRASIL. Bahia: Lençóis, Fazenda Araruna, mata semidecídua, S12 ${ }^{\circ} 17^{\prime} 6$ " e W41 ${ }^{\circ} 17^{\prime} 1$ ', 9/VI/2003, sobre folhas mortas, A.C.R. Cruz (HUEFS-56864); Palmeiras, Campos de São João, cerrado, S12 27'41" e W 4131'7”, 12/XI/2003, sobre folhas mortas, A.C.R. Cruz (HUEFS-56865).

Distribuição geográfica: Austrália (Matsushima 1989), Brasil, Índia (Grandi 1999), Costa do Marfim, Estados Unidos da América (Farr et al. 2007), Cuba (Mercado-Sierra et al. 1997), Equador (Matsushima 1993), Malásia (Kuthubutheen \& Sutton 1985), México (Heredia-Abarca 1994), Estados Federativos da Micronésia, Taiwan (Matsushima 1985), Tanzânia (Pirozynski 1968), Venezuela (Castañeda-Ruiz 2003a).

Dactylaria candidula (Höhn.) Bhatt \& Kendr., Can. J. Bot. 46: 1256. 1968.

$\equiv$ Diplorhinotrichum candidulum Höhn., Sber. Akad.

Wiss. Wien, Math.-naturw. Kl., Abt. 111: 1040. 1902.

Descrição e ilustração: Grandi (1998).
Espécie registrada pela primeira vez no Brasil por Grandi (1998). Difere das espécies relacionadas, D. uniseptata Matsush. e D. chryosperma (Sacc.) Bhatt \& Kendr. respectivamente, pela gutulação e maior dimensão dos conídios.

Material examinado: BRASIL. Bahia: Palmeiras, alto do Morro do Pai Inácio, campo rupestre, $\mathrm{S} 12^{\circ} 27^{\prime}$ e W41 ${ }^{\circ} 28^{\prime}, 10 / \mathrm{III} / 2003$, sobre folhas mortas, A.C.R. Cruz (HUEFS-80984).

Distribuição geográfica: Alemanha, Áustria, Canadá, Holanda, Inglaterra (De Hoog 1985), Brasil (Grandi 1998), Cuba (Castãneda-Ruiz 1985), Estados Unidos da América (Matsushima 1981), Japão (Matsushima 1975), Nova Zelândia (Hughes 1978), Peru (Matsushima 1993), Venezuela (Castañeda-Ruiz 2003a).

Epicoccum nigrum Link, Magazin Ges. naturf. Freunde, Berlin 7: 32. 1815.

Sinônimos ver Schol-Schwarz (1959).

Descrição e ilustração: Gusmão \& Grandi (1997).

Epicoccum nigrum é uma espécie comum, encontrada associada a diferentes substratos. ScholSchwarz (1959), em trabalho sobre o gênero e outros relacionados, salienta as variações quanto à forma dos conídios e destaca a verrucosidade, especialmente no ápice destes. Duas espécies são aceitas para o gênero, E. nigrum e E. andropogonis (Ces.) Schol-Schwarz. Epicoccum nigrum foi registrada para o Brasil associada a folhas de Alchornea triplinervia (Spreng.) Müell. Arg., Cedrela fissilis Vell., Eucalyptus sp. (Sankaran et al. 1995; Gusmão \& Grandi 1997; Grandi 1998), entre outras.

Material examinado: BRASIL. Bahia: Palmeiras, Campos de São João, cerrado, S12 $27^{\circ} 41^{\prime \prime}$ e W41 $31^{\prime} 7^{\prime \prime}, 13 / \mathrm{XI} / 2003$, sobre folhas mortas, A.C.R. Cruz (HUEFS-56842).

Distribuição geográfica: cosmopolita (ScholSchwarz 1959).

Fusariella atrovirens (Berk.) Sacc., Fung. 2: 463. 1884.

$\equiv$ Fusarium atrovirens Berk., Engl. Fl. V. p. 351. 1876. Fig. 1

Conidióforo macronematoso, mononematoso, flexuoso, septado, ramificado, hialino; célula conidiogênica enteroblástica, fialídica, terminal, integrada, determinada, verrucosa, 17-35,5 ×3,5-6 $\mu \mathrm{m}$, ápice 3,5-4,5 $\mu \mathrm{m}$; conídios em cadeia, 3-septados, levemente constritos, fusiformes, simples, curvos, lisos, 
secos, castanho-escuros, 17-24,5×4,5-6,5 $\mu$ m.

Fusariella atrovirens é facilmente reconhecida pela presença de conídios 3-septados, castanho-escuros e pela célula conidiogênica verrucosa. As características apresentadas pelos espécimes não diferiram das descritas por Ellis (1971). Entre as espécies que apresentam fiálides verrucosas, como F. aegyptiaca Mouchacca, F. indica Roy \& B. Ray e F. kansensis (Ellis \& Barth.) M.B. Ellis, o presente táxon difere, respectivamente, pela verrucosidade, menor dimensão e constrição nos conídios. Kirk et al. (2001) relatam que o gênero possui atualmente 10 espécies.

Material examinado: BRASIL. Bahia: Jussiape, caatinga, S13³5'50" e W41'42'31', 13/X/2003, sobre folhas mortas, A.C.R. Cruz (HUEFS-61605).

Distribuição geográfica: Argélia (Stalpers 2007), Europa (Ellis 1971), Índia (Farr et al. 2007).

Kiliophora ubiensis (Caneva \& Rambelli) Kuthub. \& Nawawi, Mycotaxon 48: 243. 1993.

इDanaëa ubiensis Caneva \& Rambelli, Micol. Ital. 10(1): 47. 1981.

Fig. 2-3

Conidióforo macronematoso, mononematoso, setiforme, reto ou levemente flexuoso, septado, liso, castanho-claro, 95-315×10-17 $\mu \mathrm{m}$, ápice hialino 2,5-7,5 $\mu \mathrm{m}$ larg.; célula conidiogênica enteroblástica, politrética, lateral, evidente, às vezes com proliferação percurrente, globosa, lisa, 8-10 $\mu \mathrm{m}$ diâm.; conídio solitário, 0-septado, fusiforme, simples, liso, seco, hialino, $21-25 \times 3-5 \mu \mathrm{m}$.

As características dos espécimes analisados estão de acordo com as descrições de Kuthubutheen \& Nawawi (1993), apesar da maioria das células conidiogênicas não apresentaram proliferação percurrente como relatado. O gênero é composto pela espécie descrita e por $K$. fusispora Kuthub. \& Nawawi, diferenciadas pela forma e dimensões dos conídios.

Material examinado: BRASIL. Bahia: Lençóis, Fazenda Araruna, mata semidecídua, S12 ${ }^{\circ} 17^{\prime} 6^{\prime \prime}$ e W41 ${ }^{\circ} 17^{\prime} 1$ ', 5/VI/2003, sobre folhas mortas, A.C.R. Cruz (HUEFS-56818).

Distribuição Geográfica: Brunei, Costa do Marfim, Malásia (Kuthubutheen \& Nawawi 1993).

Lauriomyces heliocephala (V. Rao \& de Hoog) Castañeda \& Kendrick, Univ. Waterloo Biol. Ser., 32: 26. 1990.

$\equiv$ Haplographium heliocephalum V. Rao \& de Hoog, Stud. Mycol. 28: 56. 1986.
Descrição e ilustração: Grandi \& Attili (1996).

Segundo Castañeda-Ruiz \& Kendrick (1990) apenas Haplographium Berk. \& Br. assemelha-se a Lauriomyces Castañeda. Estes dois gêneros possuem o mesmo tipo de conidiogênese e a coloração contrastante entre a base e o ápice do conidióforo. Entretanto, os conídios de Haplographium são agregados em mucilagem. L. pulchra Castañeda \& Kendrick poderia ser confundida com o material coletado, contudo seus conídios são maiores e clavados (Castañeda-Ruiz \& Kendrick 1990).

Material examinado: BRASIL. Bahia: Lençóis, Fazenda Araruna, mata semidecídua, S12 ${ }^{\circ} 17^{\prime} 6^{\prime \prime}$ e W41 ${ }^{\circ} 17^{\prime} 1$ ', 5/II/2003, sobre folhas mortas, A.C.R. Cruz (HUEFS-80982).

Distribuição geográfica: Brasil (Grandi \& Attili 1996), Cuba (Castañeda-Ruiz \& Kendrick 1990).

Myrothecium gramineum Libert, Pl. Crypt., Arduennae: 380. 1837.

Descrição e ilustração: Tulloch (1972).

Atualmente são aceitas 16 espécies no gênero (Kirk et al. 2001). Segundo Ellis (1971) este fungo é sapróbio abundante em ervas, e também foi isolado do solo. Maia et al. (2002) citaram o táxon pela primeira vez para o país em um levantamento da diversidade de fungos para o Estado de Pernambuco. Outras cinco espécies foram encontradas no país, $M$. prestonii M.C. Tulloch, M. leucotrichum (Peck) M.C. Tulloch, $M$. roridum Tode ex Fr., M. setiramosum Castañeda e M. verrucaria (Alb. \& Schw.) Ditm. ex Fr. (Silva \& Minter 1995; Mendes et al. 1998; L. Gusmão, dados não publicados; Castañeda-Ruiz et al. 2003b).

Material examinado: BRASIL. Bahia: Jussiape, caatinga, S13³5'50" e W41 ${ }^{\circ} 42^{\prime} 31^{\prime \prime}, 2 / \mathrm{V} / 2003$, sobre folhas mortas, A.C.R. Cruz (HUEFS-56841).

Distribuição geográfica: Brasil (Maia et al. 2002), Cuba (Mercado-Sierra et al. 1997), Escócia, Estados Unidos da América (Farr et al. 2007), Gana, Índia, Jamaica, Serra Leoa, Togo (Tulloch 1972).

Paraceratocladium silvestre Castañeda, Fungi Cubenses II 2: 9. 1987.

Fig. 4-6

Seta pontiaguda, septada, ereta, reta ou flexuosa, lisa, castanho-escura, 150-260×4,5-8 $\mu$ m; conidióforo micronematoso, mononematoso, septado, ascendendo até o ápice da seta, liso, subhialino; célula conidiogênica enteroblástica, monofialídica, lateral, evidente, reta ou curva, lisa, subhialina, 6-10,5 $\times 5,5-7,5 \mu \mathrm{m}$ larg.; colarete

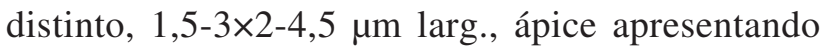


fiálides imaturas; conídio solitário, 1-septado, aciculado, levemente curvo, simples, liso, em mucilagem, subhialino, $18,5-23,5 \times 1-1,5 \mu \mathrm{m}$.

As características do espécime estudado estão de acordo com as descrições consultadas (Castañeda-Ruiz 1987; Whitton et al. 2001). No entanto, as medidas dos conídios são menores do que as relatadas por Whitton et al. (2001). O táxon está entre as espécies do gênero que apresentam setas sem ramificações, diferindo de P. bacilliformis Calduch, Stchigel, Gené \& Guarro, P. malaysianum Goh \& Hyde e $P$. triseptatum Dulym., Wu \& Peerally principalmente pela morfologia dos conídios. P. malaysianum é a espécie mais próxima, podendo ser diferenciada pelas maiores dimensões da seta e dos conídios acerosos (Goh \& Hyde 2000).

Distribuição geográfica: China (Whitton et al. 2001), Cuba (Castañeda-Ruiz 1987), México (HerediaAbarca et al. 2004).

Material examinado: BRASIL. Bahia: Lençóis, Fazenda Araruna, mata semidecídua, S12 ${ }^{\circ} 17^{\prime} 6$ " e W41 ${ }^{\circ} 17^{\prime} 1$ ', 30/V/2003, sobre folhas mortas, A.C.R. Cruz (HUEFS-61606).

Phaeoisaria infrafertilis B. Sutton \& Hodges, Nova Hedwigia 27: 219. 1976.

$\equiv$ Chryseidea africana Onofri, in Onofri, Lunghini,

Rambelli \& Lustrati, Mycotaxon 13(2): 331. 1981.

Descrição e ilustração: Grandi \& Attili (1996).

O gênero apresenta 10 espécies (Kirk et al. 2001) e $P$. infrafertilis pode ser distinguida facilmente pela forma falcada dos seus conídios e a posição mediana da região fértil (Siboe et al. 1999).

Material examinado: BRASIL. Bahia: Lençóis, Fazenda Araruna, mata semidecídua, S12 $17^{\prime} 6$ " e W41 ${ }^{\circ} 17^{\prime} 1$ ', 23/I/2004, sobre folhas mortas, A.C.R. Cruz (HUEFS-61573).

Distribuição geográfica: Brasil (Grandi \& Attili 1996), Costa do Marfim (como Chryseidea africana, Onofri et al. 1981), Ilhas Maurícios (Dulymamode et al. 2001), Quênia (Kirk 1985), Venezuela (Castañeda-Ruiz 2003a).

Pleurotheciopsis setiformis Castañeda, Deuteromycotina de Cuba, Hyphomycetes III: 28. 1985.

Fig. 7-8

Seta pontiaguda, septada, ereta, reta ou levemente flexuosa, simples, lisa, castanho-escura na base, castanho-claro no ápice, 95-180×3-6 $\mu$ m; conidióforo macronematoso, mononematoso, ereto, reto, septado, liso, castanho na base e subhialino no ápice, 30-51,5×3 $\mu \mathrm{m}$; célula conidiogênica holoblástica, poliblástica, simpodial, integrada, globosa, denticulada; conídios em cadeia curta, 1-septados, gutulados, cilíndricos, simples, secos, hialinos, 9-12,5×1,5-2,5 $\mu \mathrm{m}$.

Os espécimes analisados apresentam caracteres morfológicos de acordo com a descrição de CastañedaRuiz (1985). Contudo, os conídios do material examinado apresentaram dimensões maiores. $P$. setiformis é a única espécie desse gênero que possui setas e células conidiogênicas com desenvolvimento sincrônico, isto é, que surgem de forma simultânea (Castañeda-Ruiz et al. 2001). Essas características divergem das reportadas originalmente para o gênero, proposto por Sutton (1973), visto que as espécies por ele incluídas não possuem setas e as células conidiogênicas apresentam crescimento simpodial. Assim, Castañeda-Ruiz et al. (2001) não incluíram o táxon ora estudado em Pleurotheciopsis, sugerindo o estabelecimento de um novo gênero para acomodá-lo. Esse táxon necessita de revisão, e aqui foi aceita a proposta de Castañeda-Ruiz (1985). Trata-se, provavelmente, do segundo registro desse táxon para o mundo.

Material examinado: BRASIL. Bahia: Palmeiras, alto do Morro do Pai Inácio, campo rupestre, $S 12^{\circ} 27^{\prime}$ e W41 ${ }^{\circ} 28^{\prime}, 18 / \mathrm{II} / 2003$, sobre folhas mortas, A.C.R. Cruz (HUEFS-80976).

Distribuição geográfica: Cuba (Castañeda-Ruiz 1985).

Triscelophorus deficiens (Matsush.) Matsush., Mat. Myc. Mem. 7: 70. 1993.

$\equiv$ Triramulispora deficiens Matsush., Mat. Myc.

Mem. 3: 19. 1983.

Fig. 9

Conidióforos não observados; conídio solitário, composto por um eixo central e duas ramificações; eixo principal obclavado, truncado na base e com ápice agudo, reto, liso, seco, hialino, 15-28×1,5-2,5 $\mu \mathrm{m}$, ápice 0,5-1 $\mu \mathrm{m}$ larg., ramificação obclavada, reta ou levemente flexuosa, hialina, 10-21×1,5-2,5 $\mu \mathrm{m}$, ápice 0,5-1 $\mu \mathrm{m}$ larg.

Este táxon foi inicialmente descrito como pertencente ao gênero Triramulispora Matsush. associado a folhas e ramos em decomposição. Reexaminando o espécime, Matsushima (1993) propôs uma nova combinação, inserindo-o em Triscelophorus Ingold. Apesar de não terem sido observados conidióforos no material estudado, os conídios são típicos de T. deficiens. No entanto, as medidas da 

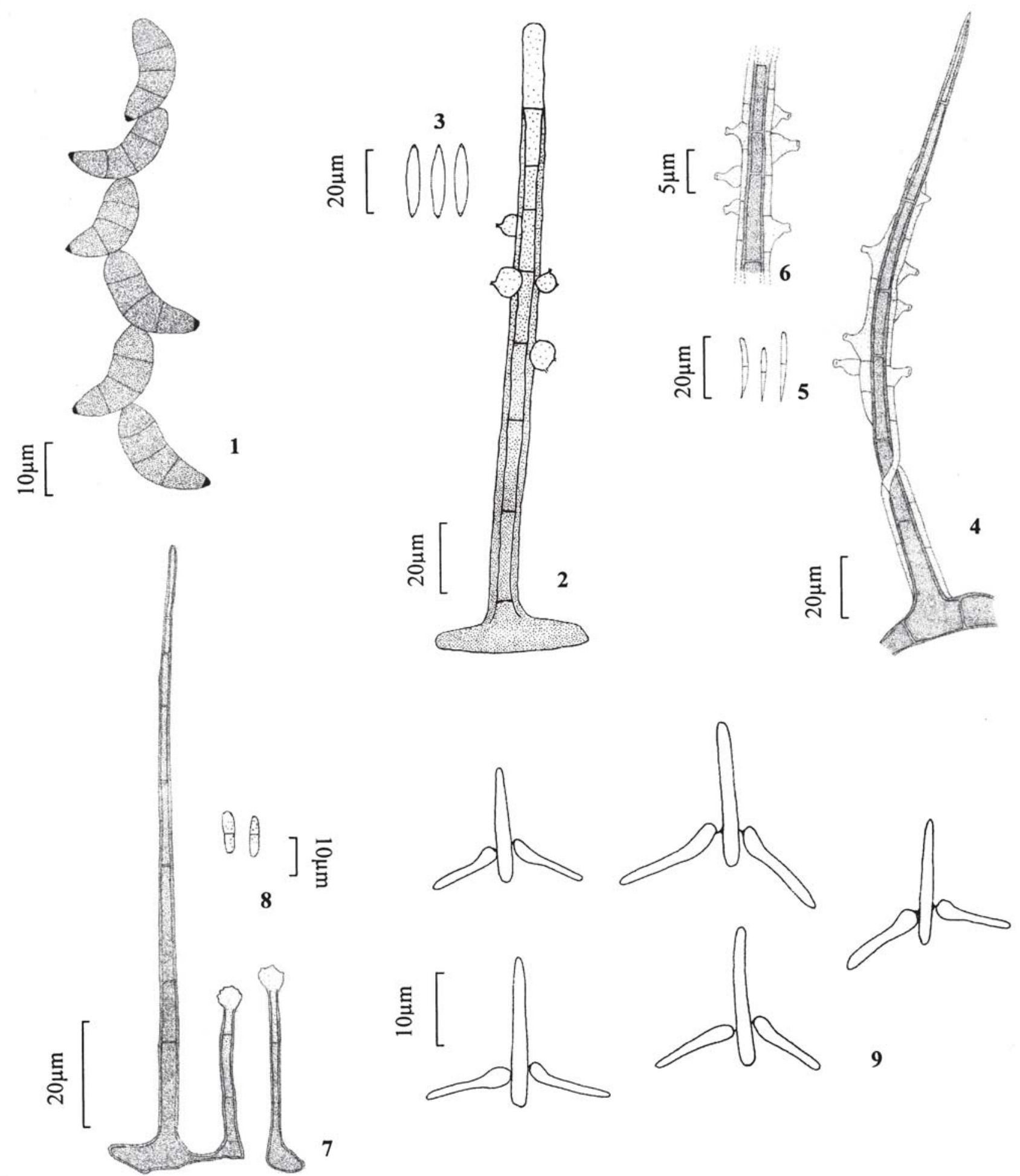

Figuras 1-9. 1. Fusariella atrovirens (Berk.) Sacc. 1. Conídios. 2-3. Kiliophora ubiensis (Caneva \& Rambelli) Kuthub. \& Nawawi. 2. Conidióforo. 3. Conídios. 4-6. Paraceratocladium silvestre Castañeda. 4. Conidióforo associado à seta. 5. Conídios. 6. Detalhe da célula conidiogênica. 7-8. Pleurotheciopsis setiformes Castañeda. 7. Conidióforo associado à seta. 8. Conídios. 9. Triscelophorus deficiens (Matsush.) Matsush. 9. Conídios. 
largura na base das ramificações foram menores que as observadas por Matsushima (1983). O gênero acomoda espécies de Hyphomycetes aquáticos que podem ocorrer tanto em folhas submersas como depositadas sobre o solo. Devido a problemas na preparação do material não foi possível observar os septos nos conídios. No Brasil já foram encontradas duas espécies, T. magnificus Petersen e T. monosporus Ingold (Schoenlein-Crusius \& Grandi 2003), as quais diferem de T. deficiens por apresentarem quatro a seis ramificações laterais em T. magnificus e três ramificações em T. monosporus.

Material examinado: BRASIL. Bahia: Lençóis, Marimbús, mata semidecídua, S12 40'9" e W41 20 '20", 6/II/2003, sobre folhas mortas, A.C.R. Cruz (HUEFS-80980a).

Distribuição geográfica: Estados Federativos da Micronésia, Estados Unidos da América, Taiwan (Matsushima 1983), Peru (Matsushima 1993).

Triramulispora gracilis Matsush., Icones Microfungorum a Matsushima llectorum (Kobe): 158. 1975.

Descrição e ilustração: Matsushima (1975).

Espécie descrita originalmente sobre folhas em decomposição, no Japão (Matsushima 1975). Para o Brasil, a espécie foi registrada pela primeira vez associada à decomposição de folhas de Miconia cabussu Hoehne (Gusmão et al. 2001). Difere da espécie próxima T. obclavata Matsush. (espécie-tipo) pela forma e tamanho das ramificações dos conídios. Problemas na preparação do material dificultaram a visualização da septação nos conídios. O fungo foi isolado juntamente com espécimens de Triscelophorus deficiens.

Material examinado: BRASIL. Bahia: Lençóis, Marimbús, mata semidecídua, S12 40'9" e W41 20 '20", 6/II/2003, sobre folhas mortas, A.C.R. Cruz (HUEFS-80980b).

Distribuição geográfica: Brasil (Gusmão et al. 2001), Japão (Matsushima 1975), México (HerediaAbarca et al. 1995).

Veronaea botryosa Cif. \& Montemart., Atti Ist. bot. Univ. Lab. crittog. Pavia, sér. 5 15: 68. 1957.

Descrição e ilustração: Matsushima (1987).

Veronaea botryosa é a espécie-tipo do gênero, sendo caracterizada por apresentar conídios cilíndricos a elipsóides (Ellis 1971; Moustafa \& Abdul-Wahid 1990). A espécie em questão difere de $V$. tectonae Kamal \& Kumar pelos conídios clavados; de
V. verrucosa Geeson, por apresentar conídios marcadamente elipsóides e verrucosos; de $V$. carlinae Ellis, por apresentar células conidiogênicas cicatrizadas e de $V$. constricta Mustafa \& Abdul-Wahid pela profunda constrição no septo (Moustafa \& AbdulWahid 1990). Montenegro et al. (1996) isolaram $V$. botryosa pela primeira vez para o Brasil em amostras de solo do município de Botucatu, Estado de São Paulo.

Material examinado: BRASIL. Bahia: Rio de Contas, cerrado, S1332'22' e W41'52'16", 21/X/2003, sobre folhas mortas, A.C.R. Cruz (HUEFS-56866); Mucugê, cerrado, S1305'51" e W41'29'6", 12/V/2003, sobre folhas mortas, A.C.R. Cruz (HUEFS-61572).

Distribuição geográfica: Brasil, China (Montenegro et al. 1996), Cuba (Mercado-Sierra \& Castañeda-Ruíz 1987), Escócia (Farr et al. 2007), Equador, Peru (Matsushima 1993), Ilhas Salomão (Matsushima 1971), Índia (Rao \& Hoog 1986), Itália (Ellis 1971), Reino Unido (Kirk \& Spooner 1984).

\section{Agradecimentos}

Os autores agradecem ao Conselho Nacional de Desenvolvimento Científico e Tecnológico (CNPq) e à Fundação de Amparo à Pesquisa do Estado da Bahia (FAPESB), pelas bolsas concedidas; ao Projeto de Conservação e Utilização Sustentável da Diversidade Biológica Brasileira (PROBIO), pelo apoio financeiro.

\section{Referências bibliográficas}

Barbosa, F.F. \& Gusmão, L.P.F. 2005. Two Speiropsis species (anamorphic fungi - Hyphomycetes) from Bahia state, Brazil. Acta Botanica Brasilica 19(3): 515-518.

Castañeda-Ruiz, R.F. 1985. Deuteromycotina de Cuba. Hyphomycetes III. Havana, Instituto de Investigaciones Fundamentales em Agricultura Tropical "Alejandro de Humboldt".

Castañeda-Ruiz, R.F. 1987. Fungi Cubenses II. Havana, Instituto de Investigaciones Fundamentales em Agricultura Tropical "Alejandro de Humboldt".

Castañeda-Ruiz, R.F.; Calduch, M.; Garcia, D. \& Izquierdo, Z. 2001. A new species of Pleurotheciopsis from leaf litter. Mycotaxon 77: 1-5.

Castañeda-Ruiz, R.F.; Gusmão, L.F.P.; Heredia-Abarca, G. \& Saikawa, M. 2006. Some hyphomycetes from Brazil. Two new species of Brachydesmiella, two new combinations for Repetophragma, and new records. Mycotaxon 95: 261-270.

Castañeda-Ruiz, R.F.; Iturriaga, T.; Minter, D.W.; Saikawa, M., Vidal, G. \& Velázquez-Noa, S. 2003a. Microfungi from Venezuela, a new species of Brachydesmiella, a new combination, and new records. Mycotaxon 85: 211-229. 
Castañeda-Ruiz, R.F.; Guarro, J.; Velásques-Noa, S. \& Gene, J. 2003b. A new species of Minimelanolocus and some Hyphomycetes records from rain forest in Brazil. Mycotaxon 85: 231-239.

Castañeda-Ruiz, R.F. \& Kendrick, B. 1990. Conidial fungi from Cuba: I. University of Waterloo Biology Series $\mathrm{n}^{\circ}$ 32: 1-53.

De Hoog, G.S. 1985. Taxonomy of the Dactylaria Complex, IV. Dactylaria, Neta, Subulispora and Scolecobasidium. Studies in Mycology 26: 1-60.

Dulymamode, R.; Cannon, P.F. \& Peerally, A. 2001. Fungi on endemic plants of Mauritius. Mycological Research 105(12): 1472-1479.

Ellis, M.B. 1971. Dematiaceous Hyphomycetes. Kew, Commonwealth Mycological Institute.

Farr, D.F.; Rossman, A.Y.; Palm, M.E. \& McCray, E.B. 2007. Fungal Databases, Systematic Botany \& Mycology Laboratory, ARS, USDA. Disponível em: http://nt.arsgrin.gov/fungaldatabases/. (Acesso: 14/janeiro/2007).

Farr, M.L. 1980. A new species of Cryptophiale from Amazonas. Mycotaxon 11: 177-181.

Gams, W. \& Holubová-Jechová, V. 1976. Chloridium and some other dematiaceous Hyphomycetes growing on decaying wood. Studies in Mycology 13: 1-99.

Giulietti, A.M. \& Pirani, J.R. 1988. Patterns of geographical distribution of some plant species from the Espinhaço Range, Minas Gerais and Bahia, Brazil. Pp. 39-69. In: P.E. Vanzolini \& W.R. Heyer (eds.). Proceedings of a Workshop on Neotropical distribution patterns. Rio de Janeiro, Academia Brasileira de Ciências.

Giulietti, A.M.; Queiroz, L.P. \& Harley, R.M. 1996. Vegetação e flora da Chapada Diamantina, Bahia. Pp. 144-156. In:

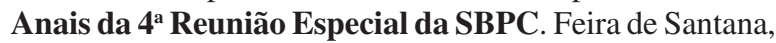
Universidade Estadual de Feira de Santana.

Goh, T.K. \& Hyde, K.D. 2000. Paraceratocladium malaysianum sp. nov from submerged wood in Malaysia. Nova Hedwigia 71: 95-100.

Grandi, R.A.P. 1998. Hyphomycetes decompositores do folhedo de Alchornea triplinervia (Spreng.) Müll. Arg. Hoehnea 25: 133-148.

Grandi, R.A.P. 1999. Hifomicetos decompositores do folhedo de Euterpe edulis Mart. Hoehnea 26: 87-101.

Grandi, R.A.P. \& Attili, D.S. 1996. Hyphomycetes on Alchornea triplinervia (Spreng.) Müell. Arg. leaf litter from the Ecological Reserve Juréia-Itatins, State of São Paulo, Brazil. Mycotaxon 60: 373-386.

Grandi, R.A.P. \& Gusmão, L.F.P. 1998. A técnica da lavagem sucessiva de substratos de plantas como subsídio para estudos da associação fungo/substrato e diversidade de Hyphomycetes nos ecossistemas. IV Simpósio de Ecossistemas Brasileiros. ACIESP 104: 80-90.

Grandi, R.A.P. \& Gusmão, L.F.P. 2002. Hyphomycetes decompositores do folhedo de Tibouchina pulchra Cogn. Revista Brasileira de Botânica 25: 79-87.

Gusmão, L.F.P. \& Barbosa, F.F. 2003. Paraceratocladium polysetosum, a new record from Brazil. Mycotaxon 96: 151-154.
Gusmão, L.F.P. \& Barbosa, F.R. 2005. Hemibeltrania (anamorphic fungi - Hyphomycetes) from Bahia state, Brazil. Sitientibus. Série Ciências Biológicas 5(1): 17-19.

Gusmão, L.F.P.; Barbosa, F.R. \& Cruz, A.C.R. 2005a. Espécies de Curvularia (fungos amamórficos - Hyphomycetes) no semi-árido do estado da Bahia. Sitientibus. Série Ciências Biológicas 5(1): 12-16.

Gusmão, L.F.P.; Góes Neto, A. \& Cruz, A.C.R. 2005b. Fungos. Pp. 227-240. In: F.A. Juncá, L. Funch \& W. Rocha (orgs.). Biodiversidade e Conservação da Chapada Diamantina. Brasília, MMA/SBF.

Gusmão, L.F.P. \& Grandi, R.A.P. 1997. Hyphomycetes com conidioma dos tipos esporodóquio e sinema associados a folhas de Cedrela fissilis (Meliaceae) em Maringá, PR, Brasil. Acta Botanica Brasilica 11: 123-134.

Gusmão, L.F.P.; Grandi, R.A.P. \& Milanez, A.I. 2000. A new species of Beltraniopsis from Brazil, with a key to the known species. Mycological Research 104: 251-253.

Gusmão, L.F.P.; Grandi, R.A.P. \& Milanez, A.I. 2001. Hyphomycetes from leaf litter of Miconia cabussu in the Brazilian Atlantic rain forest. Mycotaxon 79: 201-213.

Harley, J.L. \& Waid, J.S. 1955. A method of studying active mycelia on living roots and surfaces in the soil. Transactions of the British Mycological Society 38: 104-118.

Harley, R.M. 1995. Introduction. Pp. 1-42. In: B.L. Stannard (ed.). Flora of the Pico das Almas, Chapada Diamantina, Bahia, Brazil. Kew, Royal Botanic Gardens.

Heredia-Abarca, G. 1994. Hifomicetes dematiaceos em Bosque Mesofilo de Montaña. Registros nuevos para Mexico. Acta Botanica Mexicana 27: 15-32.

Heredia-Abarca, G.; Mercado-Sierra, A. \& Mena-Portales, J. 1995. Conidial fungi from leaf litter in a mesophilic cloud forest of Veracruz, Mexico. Mycotaxon 55: 473-490.

Heredia-Abarca, G.; Reyes-Estebanez, M. \& Arias-Mota, R.M. 2004. Adiciones al conocimiento de la diversidad de los hongos conidiales del Bosque Mesófilo de Montaña del Estado de Veracruz. Acta Botanica Mexicana 66: 1-22.

Hudson, H.J. 1968. The ecology of fungi on plant remains above the soil. New Phytopathology 67: 837-874

Hughes, S.J. 1978. New Zealand Fungi 25. Miscellaneous species. New Zealand Journal of Botany 16: 311-370.

Kirk, P.M. 1985. New or interesting microfungi XIV. Dematiaceous Hyphomycetes from MT Kenya. Mycotaxon 23: 305-352.

Kirk, P.M.; Cannon, P.F.; David, J.C. \& Stalpers, J.A. 2001. Ainsworth and Bisby's Dictionary of the fungi. $9^{\text {th }}$ ed. Wallingford, CABI.

Kirk, P.M. \& Spooner, B.M. 1984. An account of the fungi of Arran, Gigha and Kintyre. Kew Bulletin 38: 503-597.

Kuthubutheen, A.J. \& Nawawi, A. 1993. Kiliophora: a new genus name for the Hyphomycete taxon Danaea. Mycotaxon 48: 239-247.

Kuthubutheen, A.J. \& Sutton, B.C. 1985. Cryptophiale from Malaysia. Transactions of the British Mycological Society 84: 303-306. 
Maia, L.C.; Yano-Melo, A.M. \& Cavalcanti, M.A. 2002. Diversidade de fungos no Estado de Pernambuco. Pp. 15-50. In: M. Tabarelli \& J.M.C. Silva (orgs.). Diagnóstico da Biodiversidade de Pernambuco. Recife, Secretaria de Ciência, Tecnologia e Meio Ambiente. v.1.

Matsushima, T. 1971.Microfungi of the Solomon Islands and Papua-New Guinea. Published by the author. Kobe

Matsushima, T. 1975. Icones Microfungorum a Matsushima Lectorum. Published by the author. Kobe

Matsushima, T. 1981. Matsushima Mycological Memoirs n. 2. Published by the author. Kobe.

Matsushima, T. 1983. Matsushima Mycological Memoirs n. 3. Published by the author. Kobe.

Matsushima, T. 1985. Matsushima Mycological Memoirs n. 4. Published by the author. Kobe.

Matsushima, T. 1987. Matsushima Mycological Memoirs n. 5. Published by the author. Kobe.

Matsushima, T. 1989. Matsushima Mycological Memoirs n. 6. Published by the author. Kobe.

Matsushima, T. 1993. Matsushima Mycological Memoirs n. 7. Published by the author. Kobe.

Meguro, M.; Vinueza, G.N. \& Delitti, W.B.C. 1979. Ciclagem de nutrientes minerais na mata mesófila secundária - São Paulo. I - Produção e conteúdo de nutrientes minerais no folhedo. Boletim de Botânica da Universidade de São Paulo 7: 11-31.

Mendes, M.A.S.; Silva, V.L.; Dianese, J.C.; Ferreira, J.A.S.V.; Santos, C.E.N.; Gomes Neto, E.; Urden, A.F. \& Castro, C. 1998. Fungos em plantas no Brasil. Brasília, EMBRAPA, SPI.

Mercado-Sierra, A. \& Castañeda-Ruiz, R.F. 1987. Nuevos o raros hifomicetes de Cuba. I. Especies de Cacumisporium, Guedea, Rhinocladium y Veronaea. Acta Botanica Cubana 50: 1-7.

Mercado-Sierra, A.; Holubová-Jechová, V. \& Mena-Portales, J. 1997. Hifomicetos demaciáceos de Cuba, Enteroblásticos. Torino, Museo Regionale di Scienze Naturali, Monografie XIII.

Montenegro, M.R.; Miyaji, M.; Franco, M.; Nishimura, K.; Coelho, K.I.; Horie, Y.; Mendes, R.P.; Sano, A.; Fukushima, K. \& Fecchio, D. 1996. Isolation of fungi from nature in the region of Botucatu, State of São Paulo, Brazil, an endemic area of paracoccidioidomycosis. Memórias do Instituto Oswaldo Cruz 91: 665-670.
Moustafa, A.F. \& Abdul-Wahid, O.A. 1990. Veronaea constricta, a new Hyphomycete from Egyptian soils. Mycotaxon 38: 167-171.

Onofri, S.; Lughini, D.; Rambelli, A. \& Lustrati, L. 1981. New Dematiaceous Hyphomycetes from Tropical Rain Forest Litter. Mycotaxon 13: 331-338.

Pirozynski, K.A. 1968. Cryptophiale, a new genus of Hyphomycetes. Canadian Journal of Botany 46: 1123-1127.

Rao, V. \& Hoog, G.S. 1986. New or critical Hyphomycetes from India. Studies in Mycology 28: 1-84.

Sankaran, K.V.; Sutton, B.C. \& Minter, D. 1995. A checklist of fungi recorded on Eucalyptus. Mycological Papers 170: $1-376$

Schoenlein-Crusius, I.H. \& Grandi, R.A.P. 2003. The diversity of aquatic Hyphomycetes in South America. Brazilian Journal of Microbiology 34: 183-193.

Schol-Schwarz, M.B. 1959. The genus Epicoccum Link. Transactions of the British Mycological Society 42: 149-173.

Siboe, G.M.; Kirk, P.M. \& Cannon, P.F. 1999. New dematiaceous Hyphomycetes from Kenyan rare plants. Mycotaxon 73: 283-302.

Silva, M. \& Minter, D.W. 1995. Fungi from Brazil-Recorded by Batista and Co-workers. Mycological Papers 169: 1-585.

Stalpers, J. 2007. Filamentous fungi database. CBS Centraalbureau voor Schimmelcultures. Disponível em: http://www.cbs.knaw.nl/. (Acesso: 12/janeiro/ 2007).

Sutton, B.C. 1973. Some Hyphomycetes with holoblastic sympodial conidiogenous cells. Transactions of the British Mycological Society 61: 417-429.

Trappe, J.M. \& Schenck, N.C. 1982. Taxonomy of fungi forming endomycorrhizae. Pp. 1-9. In: N.C. Schenck (ed.). Methods and Principles of Mycorrhizal Research. St. Paul, The American Phytopathological Society.

Tulloch, M. 1972. The genus Myrothecium Tode ex Fr. Mycological Papers 130: 1-44.

Whitton, S.R.; McKenzie, E.H.C. \& Hyde, K.D. 2001. Microfungi on the Pandanaceae: Paraceratocladium seychellarum sp. nov. and a review of the genus. Fungal Diversity 7: 175-180. 